Recepción: 05 / 06 / 2018

Aceptación: 20 / 07 / 2018

Publicación: 15 / 09 / 2018

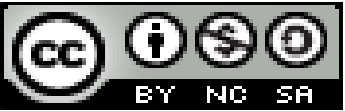

Ciencias económicas y empresariales

Artículo de investigación

\title{
Logística Comercial. Revisión Literaria
}

Commercial Logistics. Literary Review

Logística Comercial. Revisão Literária

\author{
Marcelo J. Mancheno-Saá ${ }^{\mathrm{I}}$ \\ mj.mancheno@uta.edu.ec \\ Raúl F. Villalba-Miranda II \\ raulfvillalba@uta.edu.ec \\ Jenny M. Gamboa-Salinas III \\ jgamboa12@yahoo.com \\ Juan P. Mancheno-Saá ${ }^{I V}$ \\ juanpa-007@hotmail.com
}

Correspondencia: mj.mancheno@uta.edu.ec

I Docente, Universidad Técnica de Ambato, Ambato, Ecuador.

II Magister en Gestión Estrategia Empresarial MBA, Ingeniero Comercial, Licenciado en Ciencias Administrativas, Docente de la Universidad Técnica de Ambato, Ambato, Ecuador.

III Magister en Gerencia Financiera Empresarial, Doctora en Ciencias Administrativas, Licenciada en Ciencias Administrativas, Docente de la Universidad Técnica de Ambato, Ambato, Ecuador.

${ }^{\text {IV }}$ Docente Pontificia Universidad Católica del Ecuador, Ambato, Ecuador. 


\title{
Resumen
}

El presente trabajo tuvo como objetivo revisar tendencias de investigación clásica y contemporánea en relación con la logística comercial aplicada, para ello se revisaron documentos de carácter científico, considerando los autores más representativos, donde se indagó acerca de conceptos del Término, evolución de los términos referenciales logísticos, evolución cronológica de la logística y posibles referencias al término en el entorno actual.

Es indispensable entender cómo un término que nació en el ámbito netamente militar, se ha hecho indispensable en el ámbito comercial en el siglo XXI.

Palabras clave: origen; logística; evolución logística; teoría logística; logística comercial; logística aplicada.

\begin{abstract}
The objective of this paper was to review trends in classical and contemporary research in relation to applied commercial logistics, for which purpose scientific documents were reviewed, considering the most representative authors, where they were asked about concepts of the Term, evolution of the referential terms logistics, chronological evolution of logistics and possible references to the term in the current environment.

It is essential to understand how a term that was born in the purely military field, has become indispensable in the commercial field in the XXI century.
\end{abstract}

Key words: origin; Logistics; logistic evolution; logistic theory; commercial logistics; applied logistics

\section{Resumo}

O objetivo deste trabalho foi revisar as tendências das pesquisas clássicas e contemporâneas em relação à logística comercial aplicada, para a qual foram revisados os documentos científicos, considerando os autores mais representativos, onde foram questionados sobre os conceitos do Termo, evolução dos termos referenciais logística, evolução cronológica da logística e possíveis referências ao termo no ambiente atual. 
É essencial entender como um termo que nasceu no campo puramente militar tornou-se indispensável no campo comercial no século XXI.

Palavras chave: origem; logística; evolução logística; teoria logística; logística comercial; logística aplicada.

\section{Introducción}

La palabra logística en sí, se reseña a través del vocablo logístico, que en sus primeras versiones griegas significaban calcular. (RAE, 2010) La raíz de la palabra se originó en un entorno militar en una de las potencias más recordadas del viejo mundo, la Grecia antigua. (Rico \& Mauricio, 2010). El primer término provenía del vocablo logistikos, que con el tiempo y el paso al latín por la significancia e influencia de la antigua Roma en el accionar de la época, evolucionó a logista, el cual referenciaba de gran manera al intendente de los ejércitos de aquel imperio. (Alvernia, 2006). Entonces, en un preámbulo por entender que fue la logística, cuáles son sus raíces y a que hacía referencia, simplemente el mundo contemporáneo se queda con la definición más cercana que referenciaba la organización militar en un mundo que giraba alrededor de esta. (Ballou, 1991).

Con el tiempo, la logística se diversificaría a través de una caracterización del más alto nivel, diferenciando algunas de sus actividades con cuerpo y estudio propio, entre estas se tiene a la producción, abastecimiento, transporte, movilidad y retorno. (García, 2008).

Pero no fue sino hasta el siglo 17 donde sería popularizado el término precisamente por un estudioso riguroso del entorno, el general de origen Suizo Antoine- Henri Jomini (1779-1869), su fortaleza fué la de enfocar su obra en la realización de campamentos e indicar acantonamientos sin atrincherar, un concepto que con el tiempo hizo un paralelismo en la logística comercial y que presentaba similitudes con el termino actual como la de mover hombres en la práctica y la gestión de recursos militares en el campo. (Rico \& Mauricio, 2010).

En 1870 se potencio el término, se hace referencia el ambiente de los Estados Unidos de América y Europa Occidental, que empezaba a formar una red de transporte y comunicaciones, que con el 
tiempo favorecerían el consumo. (Jameson, 1985). En esta época empezó a tomar fuerza el término de especialización. El cual también se vería vinculado con la logística.

Esta primera referencia de logística se evidencia entre los siglos XVII- XIX19, donde los ejércitos estaban tan bien entrenados en el tema de distribución, movimientos y avance, que incluso con el pasar del tiempo se han encontrado pruebas arqueológicas en lugares estratégicos como Leipzig, Alemania donde desarrollaron las Guerras Napoleónicas. (Brum, 2010).

Para (Howard, 2008), en el siguiente siglo la logística se desarrollaría en un mundo que a priori parecía civilizado, pero en el cual estallarían diferencias y darían origen a una de las épocas más oscuras del planeta, para (Alexander, 1990), las guerras mundiales indicaron a la humanidad que el término logística tendría una concepción que se haría mucho más compleja, la primera guerra mundial presentó variables básicas como la distribución de municiones, equipos y alimento.

Cada segundo contaba y por primera vez en la historia se vio que la estrategia logística podía desequilibrar los frentes en la batalla. Las variables más simples al vincularse con soldados, animales y vehículos de batalla, crearon la percepción de lo que hoy en día se conoce como la primera cadena logística. (Herwig, 2014)

En la década de los 30 se ve como la logística empieza a diferenciarse no solo a nivel militar, sino cuando esta tiene que vincularse el ámbito bélico con el de producción industrial para sustentar los diferentes conflictos, consecuencia de la ideología de la primera guerra mundial. (Herwig, 2014)

En la Segunda Guerra mundial, para (Aracil, Martí, Puigdomenech, \& Segura, 1998), la logística evolucionó de forma muy rápida debido a que cada potencia tenía su cadena de distribución y como la guerra se extendía en el tiempo la optimización de los recursos y el abasto a las tropas era primordial. La logística empresarial y logística comercial aplicada, aparecieron cuando al finalizar la guerra, los hombres que estuvieron en la guerra comenzaron a participar en el mundo comercial de la posguerra. (Los Santos, 2004)

En 1950 el mundo de posguerra, se muestra como un mundo totalmente nuevo donde la logística ha tomado un papel potencialmente protagónico, en el cual el crecimiento económico es infinito, 
en el Ecuador se consolida esta tendencia a través de la políticas de producción y de exportación bananera, haciendo que el exceso de oferta sea una de las principales características, estas características económicas marcadas, hicieron posible la autorregulación del mercado, a través del crecimiento del movimiento económico conocido como demanda. (Carvajal, 1950). Nace al mismo tiempo el concepto de satisfacción del cliente, para lo cual debe ser revisado constantemente el precio, el cual depende del costo y este a su vez de la gestión logística. (Ha, Lil, \& Ng. 2003)

En 1955, se referencia el artículo denominado "Nota sobre él", también se publicó la formulación de la Teoría de la logística de Morgenstern, en la cual los enfoques logísticos se transfirieron de contextos militares, al campo de los negocios, que en la historia contemporánea eran estudiados por primera vez. (Ruibal, 2015)

Las siguientes décadas, serían el tiempo exacto para poner a prueba el concepto logístico, para lo cual la competitividad se da a nivel de satisfacción del cliente, entra en el modelo por primera vez, la distribución física en almacenes, a lo que se suma la estrategia de gestión de inventarios, los cuales se verán documentados alrededor de 1973. (Bowerson, Closs., \& Cooper, 2002)

En la Década de los 80 con un concepto probado, aparece una época en la que se modificaría algunas preferencias al menos, así se llamó a esta etapa. La crisis de aquel momento en el sector energético, forzó la mejora de transporte y de almacenamiento, la logística en este punto tomo un enfoque modernista haciendo referencia a las preferencias de la cadena de suministro, referenciando factores específicos como proveedores, distribuidores, servicio al cliente, y haciendo proyecciones de la demanda. (Servera- Francés, 2010)

En gestión logística los inventarios, se hicieron muy reducidos tomando una concepción de competitividad en costos, los tiempos de entrega se hacen mucho más pequeños y los costos logísticos totales son acortados al máximo. (Chase, y otros, 2000). En la siguiente década, a partir del año de 1990 empiezo la época del fomento a la logística, es decir cuando las grandes empresas entienden que la optimización genera más rentabilidad, no solo se empieza hacer un uso de este término en la capacidad de las mismas, sino que se empieza a invertir para que los costos 
caigan progresivamente. Un pensamiento que en esta década va evolucionando, pero que en su esencia se mantiene igual. (Narasimhan, McLeavey, \& Billington, 1996)

Con el tiempo el término cambió, segúnn(Franco, 2016), la evolución cronológica contemporánea del término debería seguir varias pautas para poder definir el término en el entorno modernista, es así como:

1996: En este año se mencionó que el servicio que mayormente se realiza mediante la naturaleza de subcontratación es el de transporte de mercancías, los costos referenciales en USA ascendieron a 773 mil millones en 1995.

1997: La tendencia referencial de servicios en terceros aumento y las actividades que se suman a esta son las de auditoria y las de pago de facturas de mercancías.

1998: Se estableció una relación explicita entre la tecnología y la logística, indicando que la una puede potenciar a la otra.

2005: Se indicó que el know how y los fundamentos logísticos son globales.

2006: Se observó la utilización de la logística occidental en mercados emergentes como el chino.

2008: El término de cadena de suministro se relacionó con la ecología, y se establece como uno de los requisitos logísticos.

2009: La crisis y la demanda impredecible indicaron que se debía tomar en cuenta las fluctuaciones de demanda y los pronósticos de mercado.

2014: Se hicieron evidentes las necesidades de mejora en base de datos.

2015: Las herramientas tecnológicas y automatizadas pasaron a ser un pilar fundamental de la logística moderna.

Al final de la evolución histórica del termino logística se puede decir que la definición moderna parte de varios conceptos definidos militarmente como "la descendencia de la ciencia soldadesca que tiene el alistamiento, acaparamiento y manutención de los bienes de forma personal e 
instalaciones" (Suárez, 2016). En este contexto el mensaje también se usa para referir el tiempo ligado con el posicionamiento de los capitales. Con la evolución en la percepción comercial del ser humano que considera las formaciones civiles, las empresas que producen y distribuyen bienes concretos se afrontan a problemas de logística. (Los Santos, 2004)

La apreciación de varios estudiosos permitió que el significado del término "logística" se amplíe gradualmente, para incluir actividades comerciales y de servicios. (Servera - Francés, 2010). La naturaleza del término logístico, incluye aspectos como: los cuándos deben adquirirse, trasladarse y almacenarse las materias primas, los semielaborados y los productos terminados.

En las empresas en el mundo y especialmente en América Latina indican que, en el tercer mundo, la miseria de una gestión logística se presenta en dichas empresas y organizaciones públicas, que ofrecen servicios, incluidos el envío de correo, los servicios públicos y el servicio posventa. (Fernández, 2017).

Una de las organizaciones más representativas como lo es la Real Academia de la Lengua Española, ha definido al término como un conjunto de medios y métodos necesarios, para organizar una empresa o servicio, haciendo un especial énfasis en la distribución. (RAE, 2010).

La distribución comercial es un sector de actividad con importantes repercusiones sociales y económicas en los países desarrollados. Además, la distribución comercial es un instrumento o variable de marketing al igual que lo son el producto, el precio y la promoción. (Alvernia, 2006)

En la actualidad la relación entre el término logístico y las ventas se ha realizado tantas veces, que las inferencias han crecido de forma exponencial y se entiende que para que las ventas de una empresa se produzcan, no basta con tener un buen producto, a un buen precio y que sea conocido por los consumidores, sino que además, es necesario que se encuentre en el lugar y momento adecuados para que ese producto sea accesible al consumidor (Cruz, 2012).

En el entorno contemporáneo, se han diversificado las relaciones del término, siendo una de las más importantes sus relaciones con la mercadotecnia, definiéndose en un campo especifico como una herramienta del marketing contemporáneo, que relaciona la producción con las variables identificables del consumo. Además de considerar variables como la información, la promoción y 
la presentación de productos con el fin de vender una percepción y estimular al consumidor a la compra (Santemases, 2013)

Las actividades de este término universalmente aceptado y empleado han identificado varias actividades complementarias como planear, organizar, coordinar e implementar el puente de las dimensiones de tiempo y espacio dentro de un sistema. La logística es, pues, uno de las funciones más importantes de la economía según (Castellanos, 2017).

El entorno global que caracteriza al mundo empresarial, ha marcado una tendencia mundial con estrategias que van por encima de las fronteras geográficas de un país, a su vez las diferencias salariales, la expansión de los mercados extranjeros y la mejora en el transporte, rompen las barreras de tiempo-espacio entre los países y obligan a la ocupación logística a asumir una dimensión global. (Garay, 2017)

Para (Cárdenas, 2016), el mundo caritativo depende de la logística por los mismos motivos básicos que rigen los fundamentos de la mundo empresarial ligado a la obra social, los cuales se han presentado desde el inicio del comercio, para entender que también requieren de un proceso para gestionar el flujo de bienes, información y finanzas de los donantes a las personas afectadas o para solucionar la situación ameritada.

Se argumenta ampliamente que hay muchas lecciones y prácticas del mundo comercial que podrían usarse en el mundo humanitario. (CII, 2017)

Entender la comparación fundamental con el mundo comercial, radica en comprender la motivación para mejorar el proceso logístico, superando la franja de la rentabilidad y trascendiendo a lugares insospechados.

Hablando en un término holístico, que generalmente es una conjunción de varias épocas en diferentes apreciaciones, se entiende que los factores primarios a considerar son la demanda enfocada al usuario y a los pedidos, la gestión del suministro enfocada a las compras, la planificación y a la producción. Y la de cumplimiento enfocado al transporte enfocado a la distribución y al almacenamiento. (Anaya, 2015) 


\section{Métodos}

Se procedió a revisar publicaciones en Google Scholar con sus respectivos resultados en español e inglés. La revisión se la realizó para los términos que están enlazados a las palabras clave o keywords: Origen logística, Evolución logística, teoría logística, logística Comercial, logística Aplicada y sus equivalentes en inglés. El periodo evaluado se enmarca en un periodo contemporáneo desde la última década del siglo 20, mostrando un resultado de 820 referencias sobre el entorno logístico para el idioma español y 1000 para el idioma inglés.

Para tener una inferencia más objetiva de los resultados de investigación en el top 10 de cada una de los idiomas, se han tomado las 10 primeras referencias con cinco puntales estratégicos específicos en las publicaciones en las que se puede observar que en el idioma inglés.
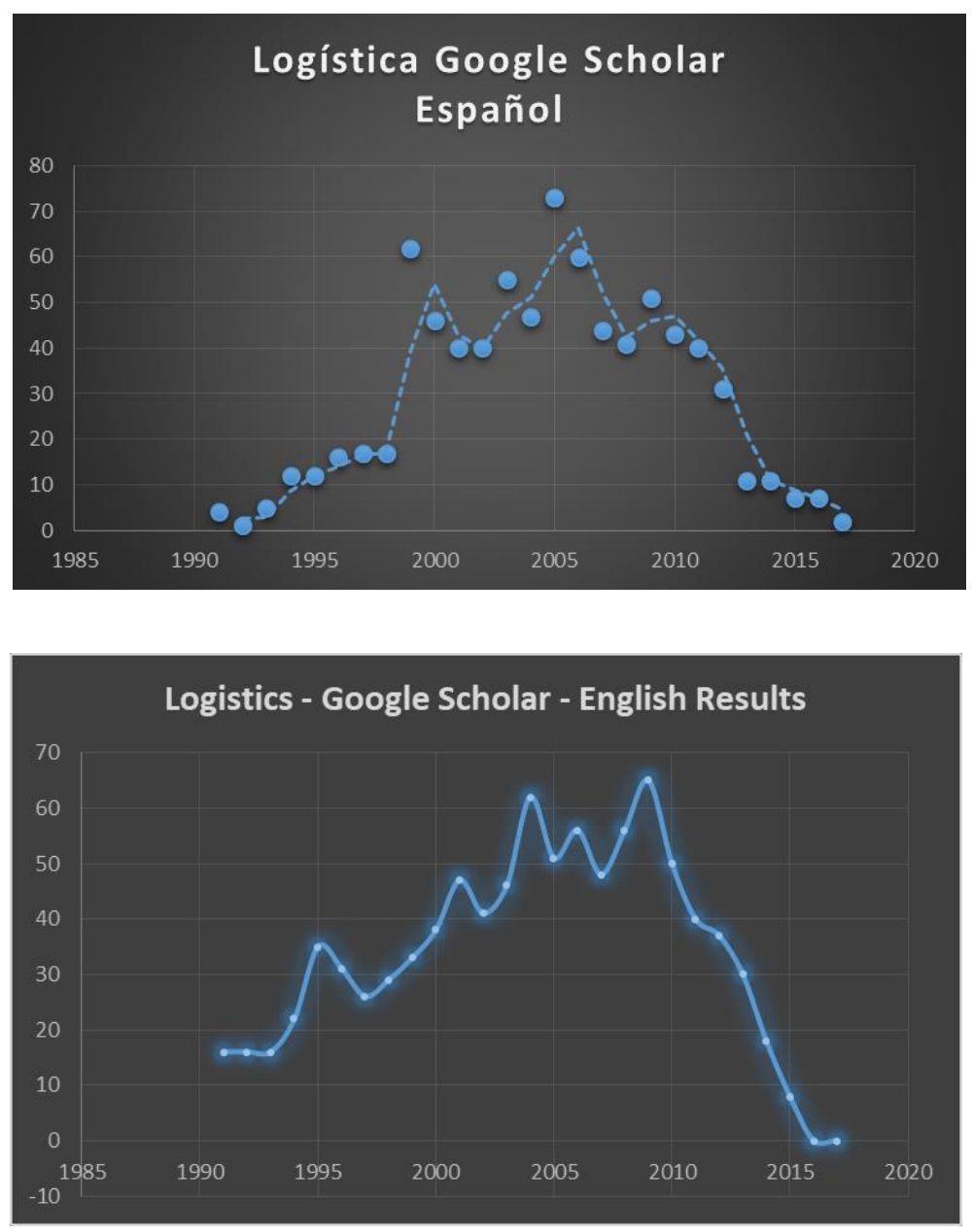


\begin{tabular}{|c|c|c|c|c|}
\hline Cites & Authors & Title & Year & Publisher \\
\hline 1535 & RH Ballou & $\begin{array}{c}\text { Gerenciamento da Cadeia de } \\
\text { Suprimentos-: Logística } \\
\text { Empresarial }\end{array}$ & 2009 & Bookman Editora \\
\hline 1535 & RH Ballou & $\begin{array}{l}\text { Logística empresarial: } \\
\text { transportes, administração de } \\
\text { materiais e distribuição física }\end{array}$ & 1993 & Atlas \\
\hline 1516 & RH Ballou & $\begin{array}{l}\text { Logística: Administración de la } \\
\text { cadena de suministro }\end{array}$ & 2004 & books.google.com \\
\hline 1470 & $\begin{array}{c}\text { DJ } \\
\text { Bowersox, } \\
\text { DJ Closs }\end{array}$ & $\begin{array}{l}\text { Logística empresarial: o } \\
\text { processo de integração da } \\
\text { cadeia de suprimento }\end{array}$ & 2007 & \\
\hline 1444 & A Novaes & $\begin{array}{l}\text { Logística e gerenciamento da } \\
\text { cadeia de distribuição }\end{array}$ & 2016 & books.google.com \\
\hline 1355 & RH Ballou & $\begin{array}{l}\text { Gerenciamento da cadeia de } \\
\text { suprimentos: planejamento, } \\
\text { organização e logística } \\
\text { empresarial }\end{array}$ & 2001 & Bookman \\
\hline 1254 & $\begin{array}{c}\text { M } \\
\text { Christopher }\end{array}$ & $\begin{array}{l}\text { Logística e gerenciamento da } \\
\text { cadeia de suprimentos: } \\
\text { estratégias para a redução de } \\
\text { custos e melhoria dos serviços }\end{array}$ & 1999 & Pioneira \\
\hline 1150 & PR Leite & $\begin{array}{l}\text { Logística reversa: meio } \\
\text { ambiente e competitividade }\end{array}$ & 2009 & $\begin{array}{c}\text { Pearson Prentice } \\
\text { Hall }\end{array}$ \\
\hline 1098 & $\begin{array}{l}\text { RH Ballou, } \\
\text { PR de } \\
\text { Lemus }\end{array}$ & $\begin{array}{c}\text { Logística empresarial: control y } \\
\text { planificación }\end{array}$ & 1991 & sidalc.net \\
\hline
\end{tabular}




\begin{tabular}{|c|c|c|c|c|}
\hline Cites & Authors & Title & Year & Source \\
\hline 162257 & $\begin{array}{l}\text { Jacob Cohen, } \\
\text { Patricia Cohen }\end{array}$ & $\begin{array}{l}\text { Applied multiple } \\
\text { regression/correlation } \\
\text { analysis for the } \\
\text { behavioral sciences }\end{array}$ & 1975 & \\
\hline 102018 & Claude E Shannon & $\begin{array}{l}\text { A mathematical theory } \\
\text { of communication }\end{array}$ & 2001 & $\begin{array}{l}\text { Mobile } \\
\text { Computing and } \\
\text { Communications } \\
\text { Review }\end{array}$ \\
\hline 70389 & Vladimir Vapnik & $\begin{array}{l}\text { Statistical learning } \\
\text { theory }\end{array}$ & 1998 & \\
\hline 67462 & $\begin{array}{l}\text { Michael C Jensen, } \\
\text { William H Meckling }\end{array}$ & $\begin{array}{l}\text { Theory of the firm: } \\
\text { Managerial behavior, } \\
\text { agency costs and } \\
\text { ownership structure }\end{array}$ & 1976 & $\begin{array}{l}\text { Journal of } \\
\text { Financial } \\
\text { Economics }\end{array}$ \\
\hline 56681 & Albert Bandura & $\begin{array}{l}\text { Self-efficacy : Toward a } \\
\text { Unifying Theory of } \\
\text { Behavioral Change }\end{array}$ & 1977 & $\begin{array}{l}\text { Psychological } \\
\text { Review }\end{array}$ \\
\hline 51382 & Icek Ajzen & $\begin{array}{l}\text { The theory of planned } \\
\text { behavior }\end{array}$ & 1991 & $\begin{array}{l}\text { Organizational } \\
\text { Behavior and } \\
\text { Human Decision } \\
\text { Processes }\end{array}$ \\
\hline 48749 & $\begin{array}{l}\text { Daniel Kahneman, } \\
\text { Amos Tversky }\end{array}$ & $\begin{array}{l}\text { Prospect Theory: An } \\
\text { Analysis of Decision } \\
\text { Under Risk }\end{array}$ & 1979 & Econométrica \\
\hline 47455 & $\begin{array}{l}\text { Thomas M Cover, } \\
\text { Joy A Thomas }\end{array}$ & $\begin{array}{l}\text { Elements of information } \\
\text { theory }\end{array}$ & 1991 & \\
\hline 46988 & Albert Bandura & $\begin{array}{l}\text { Social foundations of } \\
\text { thought and action: A } \\
\text { social cognitive theory. }\end{array}$ & 1987 & $\begin{array}{l}\text { Academy of } \\
\text { Management } \\
\text { Review }\end{array}$ \\
\hline
\end{tabular}




\section{Resultados}

Las investigaciones sobre logística y logística comercial han ido ganando importancia, aceptación y también diferentes interpretaciones del tema y su entorno. Resultó importante analizar el nivel de referencias en comparación de la base de datos en español y en inglés como muestra la siguiente figura:

\begin{tabular}{|c|c|c|}
\hline & $\begin{array}{c}\text { Citas - Frecuencia } \\
\text { Absoluta }\end{array}$ & $\begin{array}{c}\text { Frecuencia } \\
\text { Relativa }\end{array}$ \\
\hline Español & 43824 & $1 \%$ \\
\hline Inglés & 4604067 & $99 \%$ \\
\hline Total & 4647891 & $100 \%$ \\
\hline
\end{tabular}

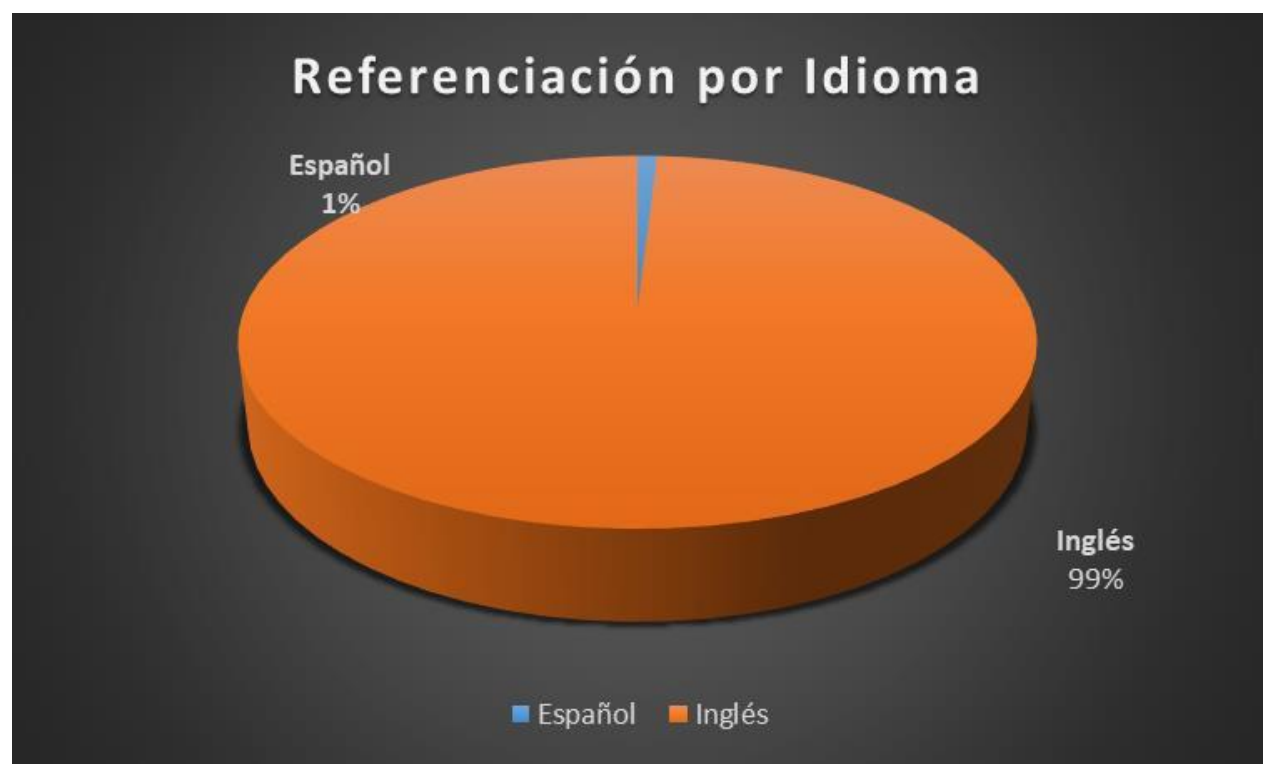

Los resultados de investigación para el término logístico en Google Scholar son mayores en el idioma inglés, a su vez, las citas son superiores, es decir la referenciarían de contenidos en investigación son 160 veces más grandes que en español y estos a su vez se han diversificado tanto en conocimiento, que se puede decir que la investigación en inglés es mayormente de carácter holístico, y también se debe considerar que esta lleva varios años de ventaja en evolución, con respecto al idioma español.

La logística indica que es un término en constante evolución, que con el pasar del tiempo incorpora varios conceptos complementarios y expande su línea de acción, sin embargo, también 
se puede observar que teorías, libros y conocimiento explicito clásico, referenciado en las décadas de los 70, 80, 90 sigue siendo útil e incluso se posiciona dentro del top 10 en referencias.

\section{El origen de un término suele estar dado por la naturaleza de sus variables y sus subvariables} haciendo referencia a los procesos de investigación realizados en la línea de tiempo, tomando como referencia solo el entorno contemporáneo - modernista:

\begin{tabular}{|c|c|c|c|}
\hline Autor & $\begin{array}{c}\text { Tema de } \\
\text { investigación }\end{array}$ & Variables principales & Variables secundarias \\
\hline $\begin{array}{c}\text { (Antún \& } \\
\text { Ojeda, 2004) }\end{array}$ & $\begin{array}{l}\text { "Benchmarking" } \\
\text { de procesos } \\
\text { logísticos }\end{array}$ & $\begin{array}{l}\text { Plan estratégico en logística, efectividad del } \\
\text { servicio al cliente, eficiencia en costos } \\
\text { logísticos, nivel de utilización de activos, } \\
\text { mejores prácticas logísticas de referencia en } \\
\text { competidores líderes o Benchmarking. }\end{array}$ & $\begin{array}{l}\text { Auditoria de logística interna y } \\
\text { externa, identificación de medidas } \\
\text { de desempeño: entradas y salidas, } \\
\text { análisis multidimensional. }\end{array}$ \\
\hline $\begin{array}{l}\text { (Ballou R. } \\
\text { 2006) }\end{array}$ & $\begin{array}{c}\text { The evolution } \\
\text { and future of } \\
\text { logistics }\end{array}$ & $\begin{array}{l}\text { Distribución, cadena de valor, previsión de la } \\
\text { demanda, planeación de compras, inventarios, } \\
\text { almacenamiento, manejo de materiales, } \\
\text { empaquetado, proceso de ordenamiento, } \\
\text { transporte, servicio al cliente, servicios de } \\
\text { información. }\end{array}$ & $\begin{array}{l}\text { Costos, flujo de la información y } \\
\text { productos, ingresos. }\end{array}$ \\
\hline $\begin{array}{l}\text { (Ballesteros } \\
\text { Riveros \& } \\
\text { Ballesteros } \\
\text { Silva, 2008) }\end{array}$ & $\begin{array}{l}\text { Importancia de la } \\
\text { administración } \\
\text { logística }\end{array}$ & $\begin{array}{l}\text { Red de distribución, decisiones logísticas por } \\
\text { niveles, control de inventarios, integración de } \\
\text { inventarios y transporte, administración del } \\
\text { equipo de transporte. }\end{array}$ & $\begin{array}{c}\text { Modelamiento de problemas } \\
\text { Logísticos, evaluación de algunas } \\
\text { técnicas de solución. }\end{array}$ \\
\hline $\begin{array}{c}\text { (Ocampo } \\
\text { Vélez, 2009) }\end{array}$ & $\begin{array}{c}\text { Gerencia } \\
\text { logística y global }\end{array}$ & $\begin{array}{l}\text { Gerencia de la cadena de abastecimiento } \\
\text { Global, Administración de la relación con los } \\
\text { proveedores, Administración de la cadena de } \\
\text { suministro interna, Administración de la } \\
\text { relación con los clientes. }\end{array}$ & $\begin{array}{l}\text { Intercambio electrónico de datos, E- } \\
\text { Business, código de barras, } \\
\text { certificaciones de calidad. }\end{array}$ \\
\hline $\begin{array}{c}\text { (Correa } \\
\text { Espinal, } \\
\text { Gómez, \& } \\
\text { Botero, 2012) }\end{array}$ & $\begin{array}{l}\text { La ingeniería de } \\
\text { métodos y } \\
\text { tiempos como } \\
\text { herramienta en la } \\
\text { cadena de } \\
\text { suministro. }\end{array}$ & $\begin{array}{l}\text { Gestión de la cadena de suministro y } \\
\text { suministros, ingeniería de métodos y tiempos, }\end{array}$ & $\begin{array}{c}\text { Flujo de información, tiempo y } \\
\text { dinero, proveedores, empresa } \\
\text { cliente. }\end{array}$ \\
\hline $\begin{array}{l}\text { (Chackelson, } \\
\text { Santos, \& } \\
\text { Errasti, 2013) }\end{array}$ & \begin{tabular}{|} 
Herramienta para \\
asistir el proceso \\
de diseño de \\
almacenes: \\
desarrollo y \\
validación.
\end{tabular} & $\begin{array}{l}\text { Demanda, diseño de almacenes, layout, } \\
\text { sistema de automatización, almacenamiento, } \\
\text { equipos de manutención, recepción, } \\
\text { ubicación, almacenaje, preparación de } \\
\text { pedidos, expedición y transporte, gestión de } \\
\text { stock, gestión de pedidos de clientes. }\end{array}$ & $\begin{array}{l}\text { Bases de datos y hojas de cálculo } \\
\text { para analizar la información, } \\
\text { software de simulación y hojas de } \\
\text { cálculo, perfil de órdenes del cliente, } \\
\text { perfil de referencias (ABC, } \\
\text { características del producto), perfil } \\
\text { del inventario, perfil de actividades }\end{array}$ \\
\hline
\end{tabular}


A nivel de logística comercial y almacenes se referencian con varios autores que han tratado de definir las investigaciones con las diferentes funciones destacadas, las cuales se enmarcan de la siguiente manera:

\begin{tabular}{|l|l|}
\hline Autor & Funciones destacadas en los almacenes \\
\hline (Anaya, 2015) & $\begin{array}{l}\text { Recepción, adecuación (embalaje, etiquetado), almacenamiento, picking, } \\
\text { preparación de la entrega, carga de camiones, entrega. }\end{array}$ \\
\hline $\begin{array}{l}\text { (Pau Cos \& De } \\
\text { Navascués, } \\
2001)\end{array}$ & $\begin{array}{l}\text { Llegada a almacén, descarga, control y manipulación, almacenaje, desalmacenaje, } \\
\text { zona de preparación, piking, embalaje, carga y transporte. }\end{array}$ \\
\hline $\begin{array}{l}\text { (De Diego } \\
\text { Morillo, 2015) }\end{array}$ & $\begin{array}{l}\text { Zona interna: Recepción, almacenaje, preparación de pedidos, expedición, oficinas } \\
\text { y actividades auxiliares, Zona externa: Transporte }\end{array}$ \\
\hline $\begin{array}{l}\text { (Antún \& } \\
\text { Ojeda, 2004) }\end{array}$ & $\begin{array}{l}\text { Servicio al cliente y procesamiento de pedidos, transporte, gestión y planeación de } \\
\text { inventarios, operaciones en almacenamiento, compras, políticas y procedimientos, }\end{array}$ \\
\hline $\begin{array}{l}\text { (Ballou R. , } \\
\text { 2004) }\end{array}$ & $\begin{array}{l}\text { Previsión de la demanda, adquisidores, inventarios, empaquetado, transporte, } \\
\text { servicio al cliente, servicios de información. }\end{array}$ \\
\hline
\end{tabular}

\section{Conclusiones}

La evolución de la logística, ha permitido que se siga referenciando dos escenarios muy lejanos, hermanos en sus inicios, el militar y el comercial, sin embargo, la evolución futura del mismo es una incógnita de relevancia que puede aperturar investigaciones en dos direcciones:

a) Los puntales estratégicos que con el tiempo saldrán de funcionamiento dentro de la funcionalidad misma del término.

b) Los puntales futuros que con el tiempo se irán identificando o a su vez se irán vinculando en el profundo estudio de este término.

\section{Los términos más representativos que hacen referencia a este término son:}
a) Proyecciones
b) Costos
c) Comportamiento 
d) Control

e) Planificación

f) Competitividad

g) Cadena de Distribución

En este análisis histórico y epistemológico de la logística comercial, en muchos aspectos y a lo largo del tiempo se relaciona estrechamente con las cadenas de suministros ya que promueven la misma misión: llevar los bienes o servicios adecuados al lugar adecuado, en el momento adecuado y en las condiciones deseadas lo cual resulta un tema de interés con mucha vigencia en la actualidad y con importantes mira al futuro de las organizaciones que mueven al mundo, pues la logística abarca todos los procesos referidos a almacenamiento, embalaje, transporte y distribución de productos así como la atención y el servicio al cliente.

Entender la importancia de la logística comercial supone la evolución y optimización de la forma de comercializar en cualquier escala, trayendo consigo importantes ventajas económicas y sociales para el mundo actual.

\section{Referencias Bibliográficas}

Alexander, J. C. (1990). Las teorías sociológicas desde la Segunda Guerra Mundial. Multimania Co.

Alvernia, E. L. (2006). La logística en su marco referencial y conceptual. Ciencia y Poder Aéreo, $1(1), 41-44$.

Aparicio, J. M. (junio de 2015). Gestión logística y comercial. Obtenido de https://www.fiuxy.bz/threads/gestion-logistica-y-comercial-1ra-edicion-juan-miguel-gomezaparicio-pdf-epub.4553387/

Aracil, R., Martí, R. A., i Puigdoménech, J. O., \& Segura, A. (1998). El mundo actual: de la Segunda Guerra Mundial a nuestros días (Vol. 2). Edicions Universitat Barcelona. 
Ballesteros, D. (2014). La logística competitiva y la administración de la cadena de suministros. Scientia Et Technica, 201-206. Obtenido de http://www.redalyc.org/html/849/84912053030/

Ballou, R. H. (2004). Logística: Administración de la cadena de suministro. Pearson Educación.

Ballou, R. H., \& de Lemus, P. R. (1991). Logística empresarial: control y planificación (No. 658 B36y.). Díaz de Santos.

Bowersox, D. J., Closs, D. J., \& Cooper, M. B. (2002). Supply chain logistics management (Vol. 2). New York, NY: McGraw-Hill.

Brum, P. (2010). Algunos comentarios sobre la Revolución Francesa. Letras Internacionales, $(111-4)$.

Cárdenas, R. L. (15 de Mayo de 2016). PLANIFICACION ESTRATEGICA DE LA

LOGISTICA. Obtenido de http://www.renelegue.cl/site/images/cont/pdf/UNIDAD_I_Planificacion_Estrategica_de_la_Logi stica.pdf

Carranza, O. (2004). Logística: mejores prácticas en Latinoamérica. Octavio Carranza.

Carvajal, F. (1950). Ecuador: la evolución de su economía 1950-2008. estado del país, 95.

Castellanos, A. (2015). Logística Comercial Internacional. Barranquilla: ECOE EDICIONES.

Castellanos, A. (2017). Logística Comercial. Colombia: EcoEdiciones.

Chase, R. B. A., Jacobs, N., Chase, R., Aquilano, N., Jacobs, R., Heizer, J., ... \& MILDRED, T. (2000). Administración de producción y operaciones: manufactura y servicios. McGraw-Hill..

Christopher, M. (2008). Logística: Aspectos estratÚgicos.

Christopher, M., \& Christopher, M. (1994). Logística y aprovisionamiento: cómo reducir costes, stocks y mejorar los servicios (No. 658.5/CH551E). 
CII. (2017). academy.connectamericas.com. Obtenido de https://academy.connectamericas.com/pluginfile.php/5369/course/summary/Transporte\%20y\%20 Logi\%CC\%81stica\%20Internacional_Syllabus.pdf

Clols, L. F. (15 de Noviembre de 2015). Scripta Nova. Obtenido de http://www.ub.edu/geocrit/sn/sn-523.pdf

Española, R. R. A. (2010). Nueva gramática de la lengua española manual. Espasa.

Fernández, R. L. (2017). Logística Comercial. México: Paraninfo.

Ferrín Gutiérrez, A. (2007). Gestión de stocks en la logística de almacenes. FC Editorial.

Franco, J. (Febrero de 2016). analdex. Obtenido de http://www.analdex.org/wpcontent/uploads/2016/02/JAVIER-FRANCO.pdf

Garay, J. (2017). Senaintro. Obtenido de https://senaintro.blackboard.com/bbcswebdav/institution/semillas/137126_1_VIRTUAL/contenid os/oaaps/oaap1/oas/oa_cadenasuministros/oc.pdf

García, L. A. (2008). Gestión logística integral. Bogotá. Ecoe Ediciones.

García, L. A. (2008). Gestión logística integral. Bogotá. Ecoe Ediciones.

García, L. A. M. (2016). Indicadores de la gestión logística. Ecoe Ediciones.

Henderson, A. (2014). El arte de elaborar el estado del arte en una investigación. Serie técnica de manuales prácticos para el investigador. Obtenido de https://repositoriotec.tec.ac.cr/bitstream/handle/2238/9145/El\%20estado\%20del\%20arte\%202017 .pdf? sequence $=1 \&$ is Allowed $=\mathrm{y}$

Hernández, R., Fernández, C., \& Baptista, P. (2012). Metodología de la investigación. México: McGraw-Hill. Obtenido de https://investigar1.files.wordpress.com/2010/05/1033525612mtis_sampieri_unidad_1-1.pdf 
Herwig, H. H. (2014). The First World War: Germany and Austria-Hungary 1914-1918. A\&C Black.

Howard, M., Furió, S., \& Howard, M. (2008). La primera guerra mundial (No. Sirsi) a458011).

https://books.google.es/books?hl=es\&lr=\&id=QIxdzTYzAFsC\&oi=fnd\&pg=PA11\&dq=indicado res+de+logistica+comercial\&ots=J8EM38ZHt9\&sig=fRzD-tdm9s-

J01q2styFtJ5ZYTE\#v=onepage \&q=indicadores\%20de\%20logistica\%20comercial\&f=false

Cruz, Ignacio (2012). Fundamentos de marketing. Barcelona: Ariel.

Jameson, F. (1985). Posmodernismo y sociedad de consumo. La posmodernidad, 165-86.

Londoño, O., Maldonado, L., \& Calderón, L. (2014). Guía para construir Estados del Arte.

Los Santos, I. S. (2004). Logística comercial y empresarial (Vol. 11). Esic Editorial.

Los Santos, I. S. (2004). Logística comercial y empresarial (Vol. 11). Esic Editorial.

Los Santos, I. S. (2006). Logística y marketing para la distribución comercial. Esic Editorial.

Santesmases, Miguel. Marketing: conceptos y estrategias (2013). Pirámide.

Real Academia Española y Asociación de Academias de la Lengua Española, Ortografía de la lengua española. Madrid: Espasa, 2010.

Rico, Á., \& Mauricio, G. (2010). El campamento militar griego: De Homero a Jenofonte.

Ruibal Handabaka, A., \& DE LA DISTRIBUCION, I. G. L. (1994). Gestión logística de la distribución física internacional.

Ruibal, A. (22 de Octubre de 2015). Palermo. Obtenido de Excelencia y desempeño en la Logística

Global: http://www.palermo.edu/economicas/mba/Pdf_09/PresentacionAlbertoRuibalHandabaka.pdf

Serrano, M. J. E. (2013). Gestión logística y comercial. Ediciones Paraninfo, SA. 
Servera-Francés, D. (2010). Concepto y evolución de la función logística. INNOVAR. Revista de Ciencias Administrativas y Sociales, 20(38).

Suárez, $\quad$ I. $\quad$ (2016). Obtenido de UNAD: https://es.scribd.com/document/339162284/FUNDAMENTOS-DE-LOGISTICA-pdf. 